Pacific Journal of Mathematic 


\title{
LOCALIZATION OF THE CORONA PROBLEM
}

\author{
T. W. GAMELIN
}

The corona problem for planar open sets $D$ and the fibers of the maximal ideal space of $H^{\circ}(D)$ are discussed and shown to depend only on the local behavior of $D$.

Let $\boldsymbol{D}$ be an open subset of the Riemann sphere $\boldsymbol{C}^{*}$, and let $\boldsymbol{H}^{\infty}(\boldsymbol{D})$ be the uniform algebra of bounded analytic functions on $D$. We will assume always that $H^{\infty}(D)$ contains a nonconstant function, that is, that $C^{*} \backslash D$ has positive analytic capacity. Our object is to study the maximal ideal space $\mathscr{C}(D)$ of $H^{\infty}(D)$, and the "fibers" $\mathscr{C}_{\lambda}(D)$ of $\mathscr{C l}(D)$ over points $\lambda \in \partial D$. The basis for our investigation is the observation that the fiber $\mathscr{M}_{\lambda}(D)$ depends only on the behavior of $D$ near $\lambda$. This localization principle is used to obtain information related to the corona problem.

The corona of $D$ is the part of $\mathscr{C}(D)$ which does not lie in the closure of $D$. Our main positive results are that $D$ has no corona under either of the following assumptions:

(1) that the diameters of the components of $C^{*} \backslash D$ (in the spherical metric, if $D$ is unbounded) be bounded away from zero; or

(2) that for some fixed $m \geqq 0$, the complement of each component of $D$ has $\leqq m$ components.

The proofs rest on the localization principle, and on Carleson's solution of the corona problem for the open unit disc [2]. Each of the above conditions includes the extension of Carleson's theorem to finitely connected planar domains due to Stout [9].

In the negative direction, we present an example, due to E. Bishop, of a connected one-dimensional analytic variety $W$ which is not dense in the maximal space of $H^{\infty}(W)$. The construction is similar to that of Rosay [8].

1. Two basic lemmas. The localization process depends on the following two lemmas.

LEMma 1.1. Let $\lambda \in \partial D$, and let $U$ be an open neighborhood of $\lambda$. If $f \in H^{\infty}(D \cap U)$, there is $F \in H^{\infty}(D)$ such that $F-f$ extends to be analytic at $\lambda$, and $(F-f)(\lambda)=0$. Moreover, $F$ can be chosen so that $\|F\|_{D} \leqq 33\|f\|_{D \cap U}$.

Indication of proof. Suppose $U=A(\lambda ; \delta)$ is the disc of radius $\delta$, centered at $\lambda$. Let $g$ be a smooth function supported on $U$, such that $g=1$ on $\Delta(\lambda ; \delta / 2)$, and $|\partial g / \partial \bar{z}| \leqq 4 / \delta$. Define $f=0$ off $D$, and set 


$$
\left(T_{g} f\right)(\zeta)=\frac{1}{\pi} \iint \frac{f(\zeta)-f(z)}{\zeta-z} \frac{\partial g}{\partial \bar{z}} d x d y .
$$

For a description of the properties of $T_{g} f$, see II.1 or VIII.10 of [3]. The desired function is obtained by adjusting $T_{g} f$ by a constant:

$$
F=T_{g} f-\frac{1}{\pi} \iint \frac{f(z)}{\lambda-z} \frac{\partial g}{\partial \bar{z}} d x d y .
$$

Lemma 1.2. Let $\lambda \in \partial D$, and let $f \in H^{\infty}(D)$. Then there is a bounded sequence $f_{n} \in H^{\infty}(D)$ such that $f_{n}$ extends to be analytic at $\lambda$, and $f_{n}(z) \rightarrow f(z)$ uniformly on any subset of $D$ at a positive distance from $\lambda$. Moreover, if $f$ extends continuously to $D \cup\{\lambda\}$, then the $f_{n}$ converge uniformly to $f$ on $D$.

Proof. This is VIII.10.8 of [3]. The proof is the same as that of 1.1, except that one uses a sequence of $g_{n}$ whose supports shrink to $\{\lambda\}$.

2. The fibers. In order to define the fibers, we prove the following lemma.

Lemma 2.1. If $\varphi \in \mathscr{M}(D)$, then there is a unique point $\lambda \in \bar{D}$ such that $\varphi(f)=f(\lambda)$ for all functions $f \in H^{\infty}(D)$ which are analytic at $\lambda$.

Proof. If $D$ is bounded, then the coordinate function $z$ belongs to $H^{\infty}(D)$, and the point $\lambda=\varphi(z)$ is easily seen to have the desired properties. Since $D$ may be unbounded, we must be more circumspect.

For convenience, we rotate the sphere so that $\infty \in D$, and so that $\varphi$ is not "evaluation at $\infty$ ". Choose $h \in H^{\infty}(D)$ such that $h(\infty)=0$ while $\varphi(h)=1$. Then $z h \in H^{\infty}(D)$. We will show that $\lambda=\varphi(z h)$ has the desired properties. Note that $\varphi((z-\lambda) h)=0$.

Suppose $f \in H^{\infty}(D)$ extends to be analytic in a neighborhood of $\lambda$. Then $(f-f(\lambda)) /(z-\lambda) \in H^{\infty}(D)$, so that $\varphi(f-f(\lambda))=\varphi(h(f-f(\lambda)))=$ $\varphi((z-\lambda) h) \varphi((f-f(\lambda)) /(z-\lambda))=0$, and $\varphi(f)=f(\lambda)$.

For the uniqueness, suppose that $\lambda^{\prime} \neq \lambda$ belongs to $\bar{D}$. We must find $F \in H^{\infty}(D)$ which is analytic at $\lambda$ and at $\lambda^{\prime}$, and which satisfies $F(\lambda) \neq F\left(\lambda^{\prime}\right)$. Using 1.2, we see that there is $f \in H^{\infty}(D)$ such that $f$ is analytic at $\lambda$ and at $\lambda^{\prime}, f(\infty)=0$, and $f$ is not identically zero on $D$. If $z_{0} \in D$ is such that $f\left(z_{0}\right) \neq 0$, then one of the three functions $f, z f,\left(f-f\left(z_{0}\right)\right) /\left(z-z_{0}\right) \in H^{\infty}(D)$ will separate $\lambda$ and $\lambda^{\prime}$. That does it.

The fiber $\mathscr{A l}_{\lambda}(D)$ of $\mathscr{M}(D)$ over $\lambda \in \bar{D}$ consists of all $\varphi \in \mathscr{M}(D)$ such that $\varphi(f)=f(\lambda)$ for all $f \in H^{\infty}(D)$ which extend to be analytic 
in a neighborhood of $\lambda$. From the definition of $\mathscr{C}_{\lambda}(D)$, and 2.1, we conclude that the $\mathscr{M}_{\lambda}(D)$ form a partition of $\mathscr{C}(D)$ into disjoint closed subsets. If $\lambda \in D$, then $\mathscr{C}_{\lambda}(D)$ consists of the single homomorphism "evaluation at $\lambda$." If $\varphi_{\alpha}$ is a net in $\mathscr{M}(D)$ converging to $\phi \in \mathscr{M}_{2}(D)$, and if $\phi_{\alpha}$ lies in the fiber over $\lambda_{\alpha}$, then the $\lambda_{\alpha}$ converge to $\lambda$.

By 1.2, the functions in $H^{\infty}(D)$ which extend analytically across $\lambda \in \partial D$ are dense in the functions in $H^{\infty}(D)$ which extend continuously to $\lambda$. We conclude the following.

Lemma 2.2. If $f \in H^{\infty}(D)$ extends continuously to $D \cup\{\lambda\}$, then $\varphi(f)=f(\lambda)$ for all $\varphi \in \mathscr{N}_{2}$.

The next theorem shows that the fibers and fiber algebras depend only on the behavior of $D$ near $\lambda$.

THEOREM 2.3. Let $\lambda \in \partial D$, and let $U$ be an open neighborhood of $\lambda$. The fibers $\mathscr{L}_{\lambda}(D)$ and $\mathscr{A}_{\lambda}(D \cap U)$ are homeomorphic. The restriction of $H^{\infty}(D)$ to $\mathscr{C}_{\lambda}(D)$ coincides (modulo this identification) with the restriction of $H^{\infty}(D \cap U)$ to $\mathscr{M}_{\lambda}(D \cap U)$.

Proof. Since $H^{\infty}(D) \subset H^{\infty}(D \cap U)$, every homomorphism in $\mathscr{C}(D \cap U)$ determines a homomorphism in $\mathscr{C}(D)$ by restricting it to $H^{\infty}(D)$. The restrictions of the homomorphisms in $\mathscr{A}_{\lambda}(D \cap U)$ belong to the fiber $\mathscr{A}_{\lambda}(D)$. This determines a continuous map of $\mathscr{A}_{\lambda}(D \cap U)$ into $\mathscr{C}_{\lambda}(D)$, which we must show is one-to-one and onto.

For this, let $\varphi \in \mathscr{M}_{2}(D)$, and $f \in H^{\infty}(D \cap U)$. Choose $F$ as in 1.1, and define $\widetilde{\varphi}(f)=\varphi(F)$. By the definition of the fiber, $\tilde{\varphi}(f)$ is independent of the function $F$, subject to the conditions of 1.1. Using 2.2 one sees that $\widetilde{\varphi}$ is multiplicative on $H^{\infty}(D \cap U)$. Moreover, if $\varphi$ is already the restriction of some $\psi \in \mathscr{A}_{2}(D \cap U)$ to $H^{\infty}(D)$, then the definition of $\widetilde{\varphi}$ shows that $\widetilde{\varphi}$ coincides with $\psi$. It follows that the correspondence $\varphi \leftrightarrow \widetilde{\varphi}$ is a homeomorphism, as was required. On account of 1.1, again, the fiber algebras are isomorphic.

COROLlaRY 2.4. With the above identification of $\mathscr{M}_{\lambda}(D)$ and $\mathscr{N}_{\lambda}(D \cap U)$, the adherence of $D$ in $\mathscr{M}_{\lambda}(D)$ coincides with the adherence of $D \cap U$ in $\mathscr{L}_{2}(D \cap U)$.

Proof. A net in $D \cap U$ will converge to $\varphi \in \mathbb{C}_{\lambda}(D)$ in $\mathscr{C}(D)$ if and only if it converges to $\tilde{\varphi} \in \mathscr{C}_{\lambda}(D \cap U)$ in $\mathscr{C}(D \cap U)$.

As another consequence of 2.3, we have the following extension of a result in [10]. 
TheOREM 2.5. The cluster set of $f \in H^{\infty}(D)$ at $\lambda \in \partial D$ coincides with the range (of the Gelfand transform) of $f$ on $\mathscr{C}_{\lambda}(D)$.

Proof. Every point in the cluster set of $f$ at $\lambda$ is assumed by $f$ on $\mathscr{L}_{\lambda}(D)$. On the other hand, suppose that $w$ does not belong to the cluster set of $f$ at $\lambda$. Then there is an open neighborhood $U$ of $\lambda$ such that $|f-w| \geqq \varepsilon>0$ on $D \cap U$. Consequently $f-w$ is invertible in $H^{\infty}(D \cap U)$, and $f$ cannot assume the value $w$ on $\mathscr{M}_{\lambda}(D \cap U)=$ $\mathscr{L}_{\lambda}(D)$.

CoRollary 2.6. If $\lambda \in \partial D$ and $f \in H^{\infty}(D)$, then

$$
\sup _{\varphi \in \mathscr{N}_{\lambda}}|\varphi(f)|=\lim _{D \ni z \rightarrow \lambda} \sup _{z \rightarrow}|f(z)| \text {. }
$$

THeOREM 2.7. The restriction $A_{\lambda}$ of $H^{\infty}(D)$ to $\mathscr{C}_{\lambda}(D)$ is a closed subalgebra of $C\left(\mathscr{C}_{2}(D)\right)$ whose maximal ideal space is $\mathscr{C}_{\lambda}(D)$.

Proof. This follows readily from the following assertion: If $h \in A_{\lambda}$, then there is $F \in H^{\infty}(D)$ such that $F=h$ on $\mathscr{C}_{\lambda}$, and $\|F\| \leqq$ $66\|h\|$. In order to establish this assertion, choose $f \in H^{\infty}(D)$ such that $f=h$ on $\mathscr{M}_{\lambda}$. By 2.6, there is an open neighborhood $U$ of $\lambda$ such that $|f| \leqq 2\|h\|$ on $D \cap U$. The desired function $F$ is now the extension of $\left.f\right|_{D \cap U}$ given by 1.1.

3. The corona problem. The open set $D$ is dense in $\mathscr{L}(D)$ if and only if whenever $f_{1}, \cdots, f_{n} \in H^{\infty}(D)$ satisfy $\left|f_{1}\right|+\cdots+\left|f_{n}\right| \geqq$ $\delta>0$ on $D$, then there exist $g_{1}, \cdots, g_{n} \in H^{\infty}(D)$ such that $f_{1} g_{1}+\cdots+$ $f_{n} g_{n}=1$. We wish to consider open sets $D$ with the following property, which is (at least formally) stronger than the assertion that $D$ be dense in $\mathscr{C}(D)$.

Property $\left(^{*}\right)$. For each integer $n \geqq 1$ and each $\delta>0$, there are constants $C(n, \delta)$ such that whenever $f_{1}, \cdots, f_{n} \in H^{\infty}(D)$ satisfy $\left|f_{j}\right| \leqq$ $1,1 \leqq j \leqq n$, and $\sum\left|f_{j}\right| \geqq \delta$ on $D$, then there exist $g_{1}, \cdots, g_{n} \in H^{\infty}(D)$ such that $\sum f_{j} g_{j}=1$ and $\left|g_{j}\right| \leqq C(n, \delta), 1 \leqq j \leqq n$.

LEMMA 3.1. An open set $D$ has the property (*) if and only if wherever $E$ is a union of disjoint open sets, each one of which is conformally equivalent to $D$, then $E$ is dense in $\mathscr{C}(E)$.

Proof. Suppose $D$ has property (*), and suppose that $f_{1}, \cdots$, $f_{n} \in H^{\infty}(E)$ satisfy $\sum\left|f_{j}\right| \geqq \delta>0$. We can assume that $\left|f_{j}\right| \leqq 1,1 \leqq$ $j \leqq n$. Using property $(*)$, we can solve the relation $\sum f_{j} g_{j}=1$ on each subset of $E$ conformally equivalent to $D$. The uniform estimate 
on the $g_{j}$ 's guarantees that the resulting solutions belong to $H^{\infty}(E)$. So $E$ is dense in $\mathscr{C}(E)$. On the other hand, if $D$ does not have property $(*)$, one easily constructs $f_{1}, \cdots, f_{n} \in H^{\infty}(E)$ such that $\sum\left|f_{j}\right| \geqq$ $\delta>0$, while $\sum f_{j} g_{j}=1$ has no analytic solutions $g_{1}, \cdots, g_{n}$ which are bounded on all of $E$.

Now Carleson [2] has shown that the open unit disc has the property $(*)$. From this, and localization, we can use a simple topological argument, as in [5], to deduce the following.

THEOREM 3.2. If the diameters (in the spherical metric) of the components of the complement of $D$ are bounded away from zero, then $D$ is dense in $\mathscr{M}(D)$.

Proof. By rotating the sphere, we can assume that $\infty \in D$. Suppose the diameters of the components $\partial D$ are bounded below by $\varepsilon>0$. If $\lambda \in \partial D$, then $D \cap \Delta(\lambda ; \varepsilon / 2)$ is simply connected, that is, each component of $D \cap \Delta(\lambda ; \varepsilon / 2)$ is conformally equivalent to a disc. By 3.1 and Carleson's theorem, $D \cap \Delta(\lambda ; \varepsilon / 2)$ is dense in $\mathscr{C}(D \cap \Delta(\lambda ; \varepsilon / 2))$. By 2.4, $\mathscr{C}_{\lambda}(D)$ belongs to the closure of $D$ in $\mathscr{C}(D)$. Since this is true for all $\lambda \in \partial D, D$ is dense in $\mathscr{C}(D)$.

The work of Behrens [1] shows that, under the hypotheses of 3.2, each fiber algebra $A_{2}$ is a logmodular algebra (on its Shilov boundary). In particular, the Gleason parts of $A_{\lambda}$ are one point parts and analytic discs. Using a Melnikov criterion (cf. [4]), it can be seen that each $\mathscr{M}_{2}$ is a peak set of $H^{\infty}(D)$, so that $\mathscr{M}_{2}$ contains every part which it meets. Hence the Gleason parts of $H^{\infty}(D)$, under the assumptions of 3.2 , are the distinct components of $D$, together with one-point parts and analytic discs.

Concerning the existence of the constants $C(n, \delta)$ for multiply connected domains, one can say the following.

THEOREM 3.3. For each choice of integers $m, n \geqq 1$, and each $\delta>0$, there exist constants $C_{m}(n, \delta)$ such that property $\left({ }^{*}\right)$ is valid, with the constants $C_{m}(n, \delta)$, for all domains $D$ which have $\leqq m$ boundary components.

Proof. Proceeding by induction, we can assume that the theorem is true, with $m$ replaced by $m-1$, so that the required constants $C_{m-1}(n, \delta)$ exist. We also assume that for some $n$ and $\delta$, the constant $C_{m}(n, \delta)$ fails to exist. From this we will obtain a contradiction.

By hypothesis, there are domains $D_{k}, 1 \leqq k<\infty$, which have $m$ boundary components, such that property $\left(^{*}\right)$ fails for $D_{k}$, with constant $C(n, \delta)=k$. We can assume that $D_{k}$ is a circle domain, obtained 
from the open unit disc $\Delta$ by excising $m-1$ disjoint closed subdiscs, one of which is centered at 0 . Let $r_{k}$ be the smallest number such that the annulus $\left\{r_{k}<|z|<1\right\}$ is contained in $D_{k}$. There will be two cases to consider: $\lim \sup r_{k}<1$ and $\lim \sup r_{k}=1$.

First, suppose that $\lim \sup r_{k}=1$. By passing to a subsequence, we can assume that $r_{k}$ converges to 1 sufficiently rapidly, so that $D_{k}$ is conformally equivalent to a domain $E_{k}$ obtained from the rectangle $\left\{2^{-k-1}<\operatorname{Im}(z)<2^{-k},-1<\operatorname{Re}(z)<1\right\}$ by excising $m-1$ holes, so that at least one of the excised holes meets $\{\operatorname{Re}(z)<-1 / 2\}$, and at least one meets $\{\operatorname{Re}(z)>1 / 2\}$. If $E=\cup E_{k}$, then the proof of 3.1 shows that $E$ cannot be dense in $\mathscr{C}(E)$. Now the open sets $E_{+}=\{z \in E$ : $\operatorname{Re}(z)>-1 / 2\}$ and $E_{-}=\{z \in E: \operatorname{Re}(z)<1 / 2\}$ are unions of domains, each of which has a complement with $\leqq m-1$ components. In view of the induction assumption, $E_{+}$and $E_{-}$are dense in $\mathscr{C}\left(E_{+}\right)$and $\mathscr{C}\left(E_{-}\right)$ respectively. If $\lambda \in \bar{E}$ satisfies $\operatorname{Re}(\lambda)>-1 / 2$, then $\mathscr{C}_{\lambda}(E)=\mathscr{C}_{\lambda}\left(E_{+}\right)$ while if $\lambda \in \bar{E}$ satisfies $\operatorname{Re}(\lambda)<1 / 2$, then $\mathscr{C}_{\lambda}(E)=\mathscr{C}_{\lambda}\left(E_{-}\right)$. In any event, every $\mathscr{C}_{\lambda}(E)$ is adherent to $E$, so that $E$ is dense in $\mathscr{C}(E)$. This contradiction allows us to reject the case $\lim \sup r_{k}=1$.

Hence we can assume that there is an $r<1$ such that each $D_{k}$ contains the annulus $\{r<|\lambda|<1\}$. Let $D$ be the disjoint union of the sets $D_{k}$, and let $H^{\infty}(D)$ be the algebra of bounded functions on $D$ which are analytic on each $D_{k}$. Again the proof of 3.1 shows that $D$ cannot be dense in the maximal ideal space $\mathscr{C}(D)$ of $H^{\infty}(D)$.

Let $N$ be the set of positive integers, and let $\Delta$ be the open unit disc. It will be convenient to regard $D$ as a subset of $\Delta \times N$, so that $H^{\infty}(\Delta \times N)$ becomes a subalgebra of $H^{\infty}(D)$. Our argument at this point is motivated by Behrens' discussion of $\mathscr{C}(\Delta \times N)$ in [1]. As Behrens notes, Carleson's theorem shows that $\Delta \times N$ is dense in $\mathscr{C}(\Delta \times N)$.

Let $\varphi \in \mathscr{C}(D)$, and let $Z$ be the function in $H^{\infty}(D)$ defined by $Z(\lambda, n)=\lambda$. We will find a net in $D$ converging to $\varphi$, and for this we consider two cases.

First, suppose that $\mid \varphi\left(Z \mid>r\right.$. The restriction $\widetilde{\varphi}$ of $\varphi$ to $H^{\infty}(\Delta \times N)$ belongs to $\mathscr{C}(\Delta \times N)$, so there is a net $\left(\lambda_{\alpha}, k_{\alpha}\right)$ in $\Delta \times N$ such that $\left(\lambda_{\alpha}, k_{\alpha}\right) \rightarrow \tilde{\varphi}$ in $\mathscr{C}(\Delta \times N)$. In other words, $f\left(\lambda_{\alpha}, k_{\alpha}\right) \rightarrow \varphi(f)$ for all $f \in H^{\infty}(D)$ which extend to be analytic on each slice $\Delta \times\{k\}, k \geqq 1$. In particular, $\lambda_{\alpha}=Z\left(\lambda_{\alpha}, k_{\alpha}\right) \rightarrow \varphi(Z)$, so that $r<\left|\lambda_{\alpha}\right|<1$ and $\left(\lambda_{\alpha}, k_{\alpha}\right) \in D$ eventually. If $F \in H^{\infty}(D)$ is arbitrary, we expand $F$ in a Laurent series, writing $F=F_{0}+F_{1}$, where $F_{0}(\lambda, k)$ is analytic on $\Delta \times\{k\}, F_{1}(\lambda, k)$ is analytic on $E_{n}=D_{n} \cup\{|\lambda| \geqq 1\}$, and $F_{1}(\infty, k)=0$. Note that $F_{0}$ and $F_{1}$ belong to $H^{\infty}(D)$, because the annuli we are splitting across have the same widths. In fact, $F_{0} \in H^{\infty}(\Delta \times N)$. Now $F_{1}(\lambda, k)=$ $F_{1}(\varphi(Z), k)+(\lambda-\varphi(Z)) H(\lambda, k)$, where $H(\cdot, k)$ is analytic on $E_{k}$. Since the distance from the boundaries of the $E_{k}$ to $\varphi(Z)$ always exceeds $|\varphi(Z)|-r$, we find that $H \in H^{\infty}(D)$. Hence 


$$
F=G+(Z-\varphi(Z)) H,
$$

where $G \in H^{\infty}(\Delta \times N)$, and $H \in H^{\infty}(D)$. Now $F\left(\lambda_{\alpha}, k_{\alpha}\right)=G\left(\lambda_{\alpha}, k_{\alpha}\right)+$ $\left(\lambda_{\alpha}-\varphi(Z)\right) H\left(\lambda_{\alpha}, k_{\alpha}\right)$ converges to $\varphi(G)=\varphi(F)$. So $\left(\lambda_{\alpha}, k_{\alpha}\right) \rightarrow \varphi$ in $\mathscr{C}(D)$, and $\varphi$ is in the closure of $D$ in $\mathscr{C}(D)$.

Next, suppose that $|\varphi(Z)| \leqq r$. The $E_{k}$ defined above are circle domains with $\leqq m-1$ holes. The induction assumption shows that if $E$ is the disjoint union of the $E_{k}$, then $E$ is dense in $\mathscr{C}(E)$. Hence there is a net $\left(\lambda_{\alpha}, k_{\alpha}\right) \in E_{k_{\alpha}} \times\left\{k_{\alpha}\right\}$ such that $f\left(\lambda_{\alpha}, k_{\alpha}\right) \rightarrow \varphi(f)$ for all $f \in H^{\infty}(D)$ which extend to be analytic on $E$. The Laurent series argument again shows that eventually $\left(\lambda_{\alpha}, k_{\alpha}\right) \in D$, and $\left(\lambda_{\alpha}, k_{\alpha}\right) \rightarrow \varphi$ in $\mathscr{C l}(D)$. Again $\varphi$ lies in the closure of $D$.

It follows that $D$ is dense in $\mathscr{C}(D)$, contradicting our previous assertion. That completes the proof of the theorem.

Now for $m, n \geqq 1$ and $\delta>0$, let $C_{m}(n, \delta)$ denote the best possible constant for which property $\left({ }^{*}\right)$ is valid for domains whose complements have $\leqq m$ components. The $C_{m}(n, \delta)$ increase with $m$. If $\sup _{m} C_{m}(n, \delta)=$ $C(n, \delta)$ is finite for all $n \geqq 1$ and $\delta>0$, then every open subset $D$ of the complex plane has property $\left({ }^{*}\right)$, with constants $C(n, \delta)$. This can be seen by approximating each component of $D$ by finitely connected domains, and using a normal families argument. If this is the case, then $D$ is dense in $\mathscr{C}(D)$ for every planar open set $D$. On the other hand, we have the following.

THEOREM 3.4. If there exist $n>1$ and $\delta>0$ such that $\sup _{m} C_{m}(n, \delta)=\infty$, then there is a domain (=connected open set) $D$ such that $D$ is not dense in $\mathscr{M}(D)$.

Proof. Suppose that for some integer $n \geqq 1$ and some $\delta>0$, there is a finitely connected domain $D_{k}$ such that property $(*)$ fails, with constant $C(n, \delta)=k$. We can assume that $D_{k}$ is contained in the rectangle $\left\{-1<\operatorname{Re}(z)<1,2^{-k-1}<\operatorname{Im}(z)<2^{-k}\right\}$, and that $\partial D_{k}$ meets both vertical sides of the rectangle. As in 3.1, $\cup D_{k}$ is not dense in $\mathscr{C}\left(\cup D_{k}\right)$. Hence there is a point $\lambda \in \partial\left(\cup D_{k}\right)$ such that $\mathscr{C l}_{\lambda}\left(\cup D_{k}\right)$ is not contained in the closure of $\cup D_{k}$. We can assume that $\operatorname{Re}(\lambda) \geqq 0$. Let $E$ be the union of $\cup D_{k}$ and the rectangle $\{-1<\operatorname{Re}(z)<-1 / 2,0<\operatorname{Im}(z)<1\}$. Then $E$ is connected, and $\mathscr{C}_{\lambda}(E)=$ $\mathscr{C}_{2}\left(\cup D_{k}\right)$. By $2.4, E$ is not dense in $\mathscr{C}(E)$. That proves the theorem.

4. An example of Bishop. Here we present an example of a one-dimensional analytic variety $W$ which is not dense in $\mathscr{C}(W)$. The example has been in circulation for some time, being originally discovered by E. Bishop some years ago, but the example has never 
appeared in print.

To construct the example, let $S$ be the shell $\{(z, w): 1 / 2<\max (|z|$, $|w|)<1\}$ in $C^{2}$. For each integer $n$, let $V_{n}$ be the set of $(z, w) \in S^{*}$ such that either $2^{n} z$ or $2^{n} w$ is a Gaussian integer. The $V_{n}$ form an increasing sequence of connected one-dimensional analytic subvarieties of $S$, whose union is dense in $S$.

Suppose $f$ is a bounded function on $U V_{n}$ which is analytic on each $V_{n}$. From Schwarz's lemma it is easy to see that $f$ is uniformly continuous, so that $f$ extends to be continuous and analytic on $S$. By Hartogs' theorem, $f$ extends to be analytic on the unit polydisc in $C^{2}$.

Lemma 4.1. There fails to exist a constant $C>0$ with the following property: For each $n$, there are $f_{n}, g_{n} \in H^{\infty}\left(V_{n}\right)$ satisfying $z f_{n}+w g_{n}=1$ and $\left|f_{n}\right| \leqq C,\left|g_{n}\right| \leqq C$.

Proof. Suppose there is such a constant. A normal families argument produces bounded functions $f$ and $g$ on $\cup V_{n}$ such that $z f+$ $w g=1$, and $f$ and $g$ are analytic on each $V_{n}$. By the remarks preceding the lemma, $f$ and $g$ extend analytically to the unit polydisc, and the extensions satisfy $z f+w g=1$. Substituting $z=w=0$, we obtain a contradiction, thereby establishing the lemma.

THEOREM 4.2. There is a connected one-dimensional analytic variety $W$ such that $H^{\infty}(W)$ separates the points of $W$, while $W$ is not dense in the maximal ideal space of $H^{\infty}(W)$.

Proof. Let $W$ be the variety obtained from the disjoint (!) union of the $V_{n}, n \geqq 2$, by identifying some prescribed point $p_{n}$ of $V_{n}$ to the point of $V_{n+1}$ with the same $z$ and $w$ coordinates, so that distinct identified pairs have distinct coordinates. Then $W$ is a connected variety, the coordinate functions $z$ and $w$ remain defined on $W$, and they satisfy $|z|+|w|>1 / 2$ on $W$. By 4.1, there fail to exist functions $f, g \in H^{\infty}(W)$ satisfying $z f+w g=1$, so that $W$ is not dense in $\mathscr{L}(W)$.

5. Extension to Riemann surfaces. It is easy to extend Lemmas 1.1 and 1.2, which allow one to localize the fibers and fiber algebras, to domains on a finite bordered Riemann surface. More specifically, we can easily handle the following situation.

Let $D$ be an open set on a Riemann surface $S$, let $\lambda \in \partial D$, and let $U$ be an open coordinate disc centered at $\lambda$. Suppose there is a function $h$ meromorphic on $D \cup U$ such that $h(\lambda)=0, h^{-1}(h(U))=U$, and $H$ is a one-to-one covering of $U$ over $h(U)$. If $f \in H^{\infty}(D \cap U)$, 
then $f \circ h^{-1} \in H^{\infty}(h(D) \cap h(U))$. By 1.1, there is a function $G \in H^{\infty}(h(D))$ such that $G-f \circ h^{-1}$ is analytic at 0 and vanishes there. Then $G \circ h=$ $F \in H^{\infty}(D)$, and $F-f$ is analytic at $\lambda$ and vanishes there. So Lemma 1.1 is valid. Also, Lemma 1.2 is valid. If the fiber $\mathscr{A l}_{2}(D)$ is defined as in $\S 2$, then 2.2 and the localization Theorem 2.3 are true.

Now suppose $D$ is a domain on a finite bordered Riemann surface. It is easy to see, using meromorphic functions, that 2.1 is valid, that is, that $\mathscr{C}(D)$ can be partitioned into disjoint closed "fibers" $\mathscr{C}_{\lambda}(D)$ over points $\lambda \in \bar{D}$. In this case, the required function $h$ always exists, for any point $\lambda \in \partial D$, so that the fibers are local. In particular, if $D$ is a finite bordered Riemann surface, then $D$ is dense in $\mathscr{C}(D)$, and the fibers and fiber algebras associated with points of $\partial D$ are identical to those associated with the disc algebra $H^{\infty}(\Delta)$. This latter theorem has been proved in a variety of ways in the literature. For one of the simplest proofs, see [7].

If $D$ is an open set lying on a compact Riemann surface, such that $H^{\infty}(D)$ contains a nonconstant analytic function, and if the fibers $\mathscr{C}_{\lambda}(D)$ are defined as in $\S 2$, then again the $\mathscr{C}_{\lambda}(D), \lambda \in \bar{D}$, form a partition of $\mathscr{C}(D)$ into disjoint closed subsets, and the localization Theorem 2.3 is valid. The details of the proofs are left.

\section{REFERENCES}

1. M. Behrens, On the corona problem for a class of infinitely connected domains, Bull. Amer. Math. Soc. 76 (1970), 387-391.

2. L. Carleson, Interpolation by bounded analytic functions and the corona problem, Ann. of Math. 76 (1962), 542-559.

3. T. Gamelin, Uniform algebras, Prentice-Hall, Englewood Cliffs, N. J., 1969.

4. T. Gamelin and J. Garnett, Distinguished homomorphisms and fiber algebras, Amer.

J. Math. (to appear).

5. J. Garnett, On a theorem of Mergelyan, Pacific J. Math. 26 (1968), 461-467.

6. K. Hoffman, Banach spaces of analytic functions, Prentice-Hall, Englewood Cliffs, N. J., 1969.

7. - Bounded analytic functions and Gleason parts, Ann. of Math. 86 (1967), 74-111.

8. J.-P. Rosay, Sur un problème posé par W. Rudin, C. R. Acad. Sci. Paris 267 (1968), 922-925.

9. E. Stout, Two theorems concerning functions holomorphic on multiply connected domains, Bull. Amer. Math. Soc. 69 (1963), 527-530.

10. L. Zalcman, Bounded analytic functions on domains of infinite connectivity, Trans. Amer. Math. Soc. 144 (1969), 241-269.

Received May 26, 1969.

University of California, Los ANgeles 



\section{PACIFIC JOURNAL OF MATHEMATICS}

EDITORS

H. SAMELSON

Stanford University

Stanford, California 94305

RichaRd PIERCE

University of Washington

Seattle, Washington 98105
J. DUGUNDJI

Department of Mathematics

University of Southern California

Los Angeles, California 90007

RICHARD ARENS

University of California

Los Angeles, California 90024

\section{ASSOCIATE EDITORS}

E. F. BECKENBACH

B. H. NeumanN

F. WOLF

K. YOSHIDA

\section{SUPPORTING INSTITUTIONS}

UNIVERSITY OF BRITISH COLUMBIA

CALIFORNIA INSTITUTE OF TECHNOLOGY

UNIVERSITY OF CALIFORNIA

MONTANA STATE UNIVERSITY

UNIVERSITY OF NEVADA

NEW MEXICO STATE UNIVERSITY

OREGON STATE UNIVERSITY

UNIVERSITY OF OREGON

OSAKA UNIVERSITY

UNIVERSITY OF SOUTHERN CALIFORNIA
STANFORD UNIVERSITY

UNIVERSITY OF TOKYO

UNIVERSITY OF UTAH

WASHINGTON STATE UNIVERSITY

UNIVERSITY OF WASHINGTON

AMERICAN MATHEMATICAL SOCIETY CHEVRON RESEARCH CORPORATION TRW SYSTEMS

NAVAL WEAPONS CENTER 


\section{Pacific Journal of Mathematics}

May, 1970

Johan Aarnes, Edward George Effros and Ole A. Nielsen, Locally compact

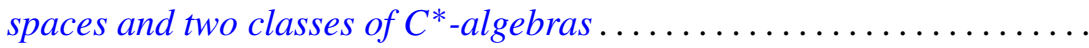

Allan C. Cochran, R. Keown and C. R. Williams, On a class of topological

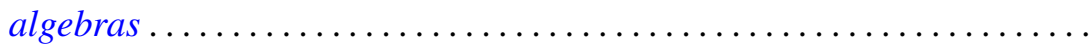

John Dauns, Integral domains that are not embeddable in division rings ....

Robert Jay Daverman, On the number of nonpiercing points in certain

crumpled cubes.....................................

Bryce L. Elkins, Characterization of separable ideals ................

Zbigniew Fiedorowicz, A comparison of two naturally arising uniformities

on a class of pseudo-PM spaces ...........................

Henry Charles Finlayson, Approximation of Wiener integrals of functionals

continuous in the uniform topology ........................

Theodore William Gamelin, Localization of the corona problem ...........

Alfred Gray and Paul Stephen Green, Sphere transitive structures and the

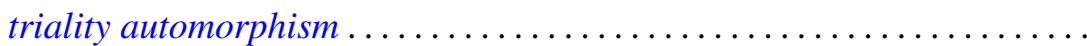

Charles Lemuel Hagopian, On generalized forms of aposyndesis ..........

J. Jakubík, On subgroups of a pseudo lattice ordered group ...............

Cornelius W. Onneweer, On uniform convergence for Walsh-Fourier

series..................................

Stanley Joel Osher, On certain Toeplitz operators in two variables ...

Washek (Vaclav) Frantisek Pfeffer and John Benson Wilbur, On the

measurability of Perron integrable functions............

Frank J. Polansky, On the conformal mapping of variable regions...

Kouei Sekigawa and Shûkichi Tanno, Sufficient conditions for a Riemannian manifold to be locally symmetric ...................

James Wilson Stepp, Locally compact Clifford semigroups ....

Ernest Lester Stitzinger, Frattini subalgebras of a class of solvable Lie

algebras ................................

George Szeto, The group character and split group algebras...

Mark Lawrence Teply, Homological dimension and splitting torsion

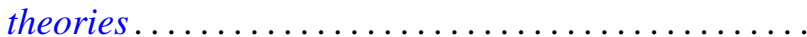

David Bertram Wales, Finite linear groups of degree seven. II ...

Robert Breckenridge Warfield, Jr., An isomorphic refinement theorem for

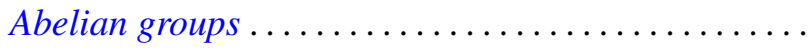

James Edward West, The ambient homeomorphy of an incomplete subspace

of infinite-dimensional Hilbert spaces................

Peter Wilker, Adjoint product and hom functors in general topology ...

Daniel Eliot Wulbert, A note on the characterization of conditional 